\title{
Analysis methodology and recent results of the IGS network combination
}

\author{
R. Ferland, J. Kouba, and D. Hutchison \\ Geodetic Surveys Division, Natural Resources Canada, 456-615 Booth Street, Ottawa, Canada K1A 0E9
}

(Received January 13, 2000; Revised August 31, 2000; Accepted September 2, 2000)

\begin{abstract}
A working group of the International GPS Service (IGS) was created to look after Reference Frame (RF) issues and contribute to the densification and improvement of the International Terrestrial Reference Frame (ITRF). One important objective of the Reference Frame Working Group is to generate consistent IGS station coordinates and velocities, Earth Rotation Parameters (ERP) and geocenter estimates along with the appropriate covariance information. These parameters have a direct impact on other IGS products such as the estimation of GPS satellite ephemerides, as well as satellite and station clocks. The information required is available weekly from the Analysis Centers (AC) (cod, emr, esa, gfz, jpl, ngs, sio) and from the Global Network Associate Analysis Centers (GNAAC) (JPL, mit, ncl) using a "Software Independent Exchange Format" (SINEX). The AC are also contributing daily ERPs as part of their weekly submission. The procedure in place simultaneously combines the weekly station coordinates, geocenter and daily ERP estimates. A cumulative solution containing station coordinates and velocity is also updated with each weekly combination. This provides a convenient way to closely monitor the quality of the estimated station coordinates and to have an up to date cumulative solution available at all times. To provide some necessary redundancy, the weekly station coordinates solution is compared against the GNAAC solutions. Each of the 3 GNAAC uses its own software, allowing independent verification of the combination process. The RMS of the coordinate differences in the north, east and up components between the AC/GNAAC and the ITRF97 Reference Frame Stations are 4-10 mm, 5-20 mm and 6-25 mm. The station velocities within continental plates are compared to the NNR-NUVEL1A plate motion model (DeMets et al., 1994). The north, east and up velocity RMS are $2 \mathrm{~mm} / \mathrm{y}, 3 \mathrm{~mm} / \mathrm{y}$ and $8 \mathrm{~mm} / \mathrm{y}$. Note that NNR-NUVEL1A assumes a zero vertical velocity.
\end{abstract}

\section{Reference Frame Stations}

A set of so called RF stations is used to align the weekly and cumulative solutions. For the IGS realization of ITRF97, the RF station set is composed of 51 carefully selected stations. Criterion such as monumentation, data quality, data latency, collocation with other techniques, accurate velocity estimate, geographical distribution etc. were used for the station selection (Kouba et al., 1998). Since GPS week 1021 (August 1, 1999), the weekly and cumulative solutions are aligned to ITRF97 (Boucher et al., 1999, http: / / lareg.ensg. ign.fr/ITRF). The IGS (http://igscb.jpl.nasa. gov) realization of ITRF96 used 47 stations; most are in the current realization. The earlier realizations used only 13 stations; most of those are still being used in the current realization. The limited number of stations in the early realizations was occasionally causing instabilities, especially when several stations at crucial locations were missing. The larger number of stations in the more recent realizations greatly reduces those potential instabilities. The weekly and the cumulative solutions are aligned to ITRF and include complete covariance matrices, as well as auxiliary station information such as receiver and antenna type, antenna offset, dome number, etc.

The weekly submissions of the preliminary RF products started with GPS week 0999. Work is continuing to improve

Copy right (C) The Society of Geomagnetism and Earth, Planetary and Space Sciences (SGEPSS); The Seismological Society of Japan; The Volcanological Society of Japan; The Geodetic Society of Japan; The Japanese Society for Planetary Sciences. the quality and timeliness of the submissions. The cumulative solution includes GNAAC solutions dating back to GPS week 0837. Starting with GPS week 0978 the AC were included in the combination while the GNAAC were used to quality control the combination. All AC use their own software, except for emr and jpl which use the GIPSY software. Although the combination was only made available starting with GPS week 0999, the procedure was tested on weekly solutions dating back to GPS week 0978.

\section{Weekly Combination Methodology}

To meet the working group objectives, a semi-automated procedure was put in place to do the weekly combinations. The procedure performs the following functions for the input solutions: 1) validate; 2) unconstrain; 3) transform to the current ITRF; 4) compare; 5) combine and 6) report. The combination is presently generated within 2 days after the last GNAAC is available. The algorithms used follow generally accepted geodesy procedures (Vanicek and Krakiwsky, 1986).

\subsection{Validation}

The format validation ensures that all the files used respect the SINEX V 1.0 (ftp: / / igscb.jpl.nasa.gov/pub/ data/format/sinex.txt). During the validation process, changes are also made such that all the SINEX files use a consistent interpretation of the SINEX format. This implies that minor differences may exist between the input $\mathrm{AC}$ and GNAAC information provided and the reported information. 
The site names and point are changed to be consistent with the official "igs.snx" file. Corrections to the parameters may also be applied if they can be justified (e.g.: "igs.snx", station logs, weekly reports, etc.). The "igs.snx" file contains a summary of the station logs and is maintained at the IGS central bureau. Corrections for pole tide and the addition of the short-term effects to the excess of Length of Day (LOD) are applied when appropriate. LOD bias corrections, based on the IERS Bulletin A, are also applied. The ERPs are always referred to the ITRF origin.

\subsection{Unconstraining}

All the AC weekly estimated solutions have their station coordinates constrained to the reference frame, currently ITRF97. The applied constraints are reported with each solution. To extract each solution normal equations needed in the combination process, the solutions are unconstrained using apriori information provided in the SINEX solution. The possibility of distortions caused by the apriori information is also eliminated. Occasional difficulties with unconstraining or inverting matrices appear to be caused mainly by rounding/truncation problems. Those are fairly minor and are resolved by simply rescaling the estimated and/or apriori diagonal matrix. The diagonal matrix rescaling is usually well below one part per million. All the weekly matrices are also rescaled by a variance factor $\left(\mathrm{Chi}^{* *} 2 /\right.$ (degrees of freedom) determined during a comparison with the combined cumulative solution. The applied scale factors are reported.

Some solutions do contain multiple estimates for a given point at a site. Within a solution they are usually recombined. The coordinate differences between those multiple solutions are generally within a few $\mathrm{mm}$. In the situation where significant differences exist, the outlier is rejected. All the AC solutions have their geocenter implicitly at the origin. An explicit geocenter is added to the parameters with coordinates $(0.0,0.0,0.0)$ for each AC SINEX file.

\subsection{Transformation}

The alignment of all the unconstrained weekly solutions to ITRF is done with a 7-parameters (3 translations, 3 rotations and 1 scale) similarity transformation. The ERPs are corrected by the appropriate transformation rotation angles and are always referred to the origin. The transformation parameters are reported. All the common points between each weekly solution and the RF stations are used to estimate the transformation. Unit weighting is used for the coordinates during the estimation of the transformation parameters. The use of the corresponding weight matrices usually leads to very similar transformation parameters. Occasionally, the transformation has shown to be sensitive to the matrices. Since the process is to be run as automatically as possible, the more robust unweighted estimation was chosen for the transformation. To ensure proper alignment to ITRF, the process also checks and deletes outliers. The outlier detection threshold is currently set at 5 sigmas. Any station deleted is reported with the residuals and the solutions involved.

\subsection{Comparison}

In an effort to produce reliable weekly and updated cumulative solutions, several comparisons are made to detect and reject outliers. The AC/GNAAC are compared with each other, with the RF stations and with the previous week "weekly" and cumulative solutions. During the comparison, it is assumed that the ITRF RF stations, the previous week "weekly" and the cumulative solutions are correct. Those are reasonable assumptions, because, several independent groups have carefully checked the ITRF RF stations; and the previous week "weekly" and cumulative solutions were also checked during the previous week combination. The weighted average and the RMS of the station coordinates residuals between the AC/GNAAC and 1) the ITRF RF stations, and 2) the weekly combination and 3) the cumulative combination are reported. Results of those comparisons will be presented below. Weighted average and RMS statistics are also reported for the daily ERP's between the AC/GNAAC and the weekly combinations.

A pair-wise comparison between the weekly $\mathrm{AC}$ and GNAAC ensures that they are consistent. Detected outliers are by default rejected in both files, except when there are indications that only one station solution is responsible. This process reveals station coordinates with significant inconsistencies. The weekly AC and GNAAC solutions are also compared with the previous week combined solution to detect significant station coordinates variations between consecutive weeks. The outlier stations are rejected from the offending solutions. This comparison detects significant station coordinates variations from week to week. The weekly AC and GNAAC solutions are finally compared with the cumulative solution to detect outliers in the station coordinates time series. Any detected/rejected outlier is again reported. When stations are rejected, it often occurs at several of the input solutions in which they are present. They usually have similar residuals and statistics; which is a good indication of the estimates and covariances consistency. This indicates that the anomalous variations are likely contained in or due to the corresponding RINEX observations.

\subsection{Combination}

The weekly AC solutions are combined to produce the two weekly combinations, the "weekly" and cumulative. The full covariance information is used to combine the station coordinates, geocenter and daily ERP. Although, no rejection is expected from the combination, the outlier detection/rejection/reporting process is repeated. The cumulative solution, which also includes station velocity estimates, is then updated. It is followed by one last outlier detection/rejection/reporting exercise. The combined "weekly" and cumulative solutions are aligned to ITRF using respectively 7 or 14 transformation parameters. The station coordinates and ERP consistency is maintained during the transformation. The results are checked and the process is repeated if necessary. The SINEX files consistency with the "igs.snx" template is also ensured. A summary report is prepared.

\subsection{Report}

The weekly combination report is divided in 5 sections: 1) Contacts; 2) Products; 3 ) Combination Strategy; 4) Remarks and 5) Results.

The product section lists the files generated each week. They are available from NASA Crustal Dynamics Data Information System (CDDIS), which is used for the archival of space geodetic data (ftp: / / cddisa.gsfc.nasa.gov/ gps/products/wwww). There are two SINEX combined solution files, i.e. "weekly" and cumulative (igsyyPwwww. snx, IGSyyPww.snx), three residual files (igsyyPwwww. 
ACs \& GNAACs Scale Factor

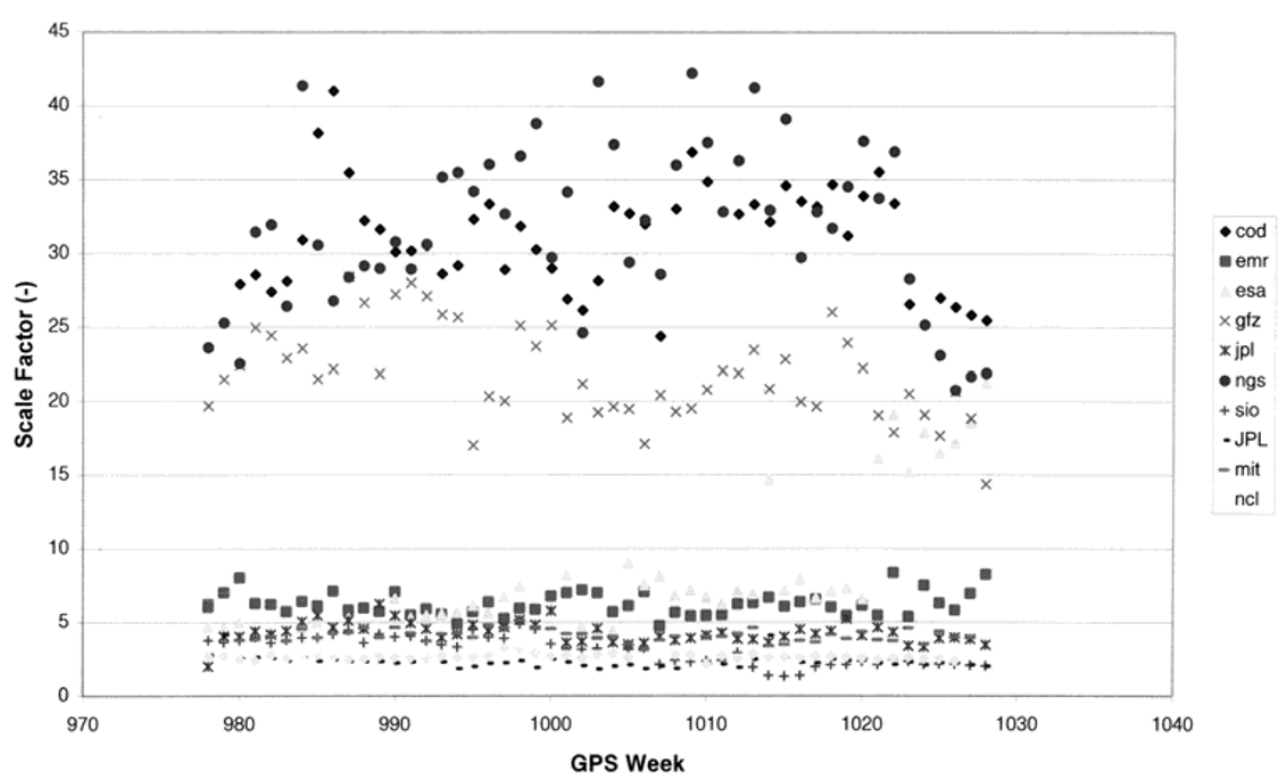

Fig. 1. Estimated Scale Factors (square root of variance factor for the AC and the GNAAC from GPS weeks 0978 (98/10/04) to 1028 (99/09/25).

ITRF97 minus AC \& GNAAC Reference Frame Station Coordinates RMS [ GPS weeks $0978(98 / 10 / 04)$ - $1028(99 / 09 / 25)$ ]
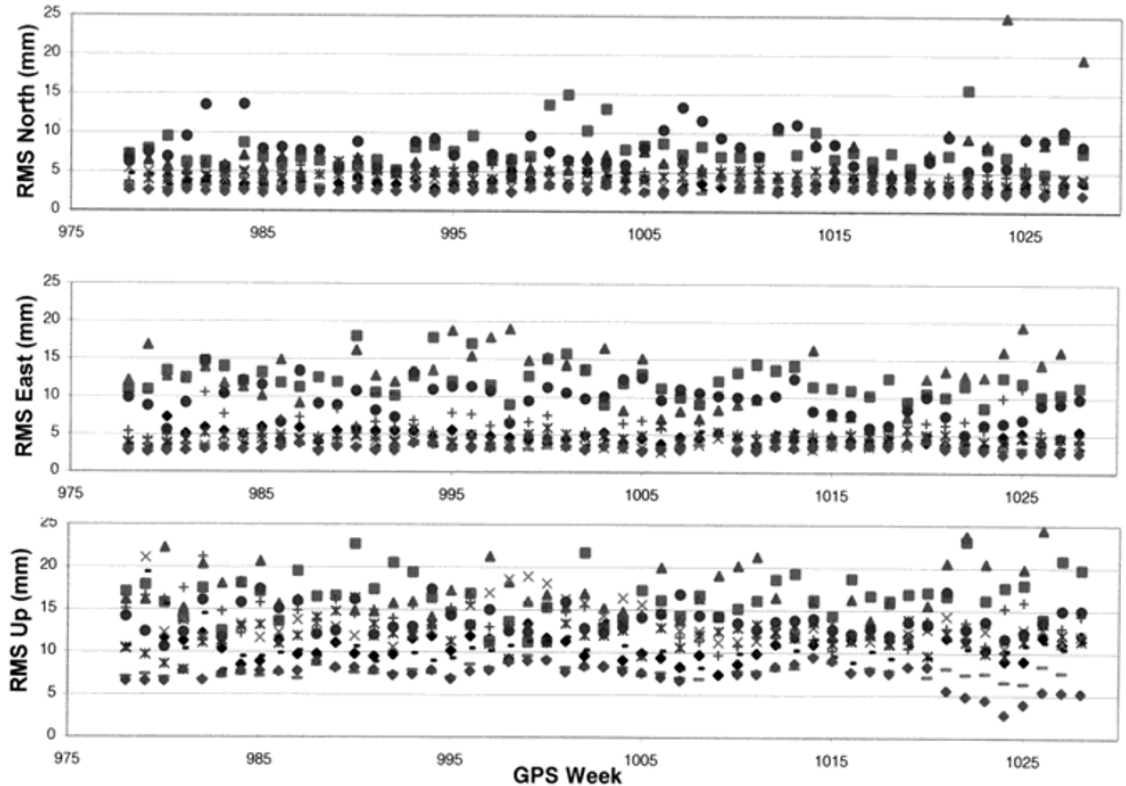

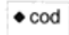

- emr

$\Delta$ esa

$\times$ gtz

x.jpl

$-n g s$
+ sio

- JPI

- mit

$\bullet$ ncl

Fig. 2. RMS North/East/Up Station Coordinate differences between the AC and GNAAC and ITRF for the Reference Frame Stations.

itr, igsyyPwwww.res, IGSyyPww.res), one ERP file (igsyyPwwww.erp) and one summary file (igsyyPwwww. sum); where "yy" are the last two digits of the year, "ww" is the week of the year and "wwww" is the GPS week. The three residual files list the station residuals with respect to the ITRF RF stations, the weekly solution and the cumulative solution. In the case of the "weekly" combined solution, the residuals are also given for the ERPs and the geocen- ter. Some remarks may also be included to clarify some information and/or action taken during the weekly combination process. The results section presents a summary of the combination process. It is divided in 7 sub-sections: 1) the variance factor, 2) the stations residuals weighted average and RMS; 3 ) the 7-parameter transformations for the RF stations; 4) the geocenter; 5) the ERP residuals weighted average and RMS as is 6) the combination/comparison outliers 

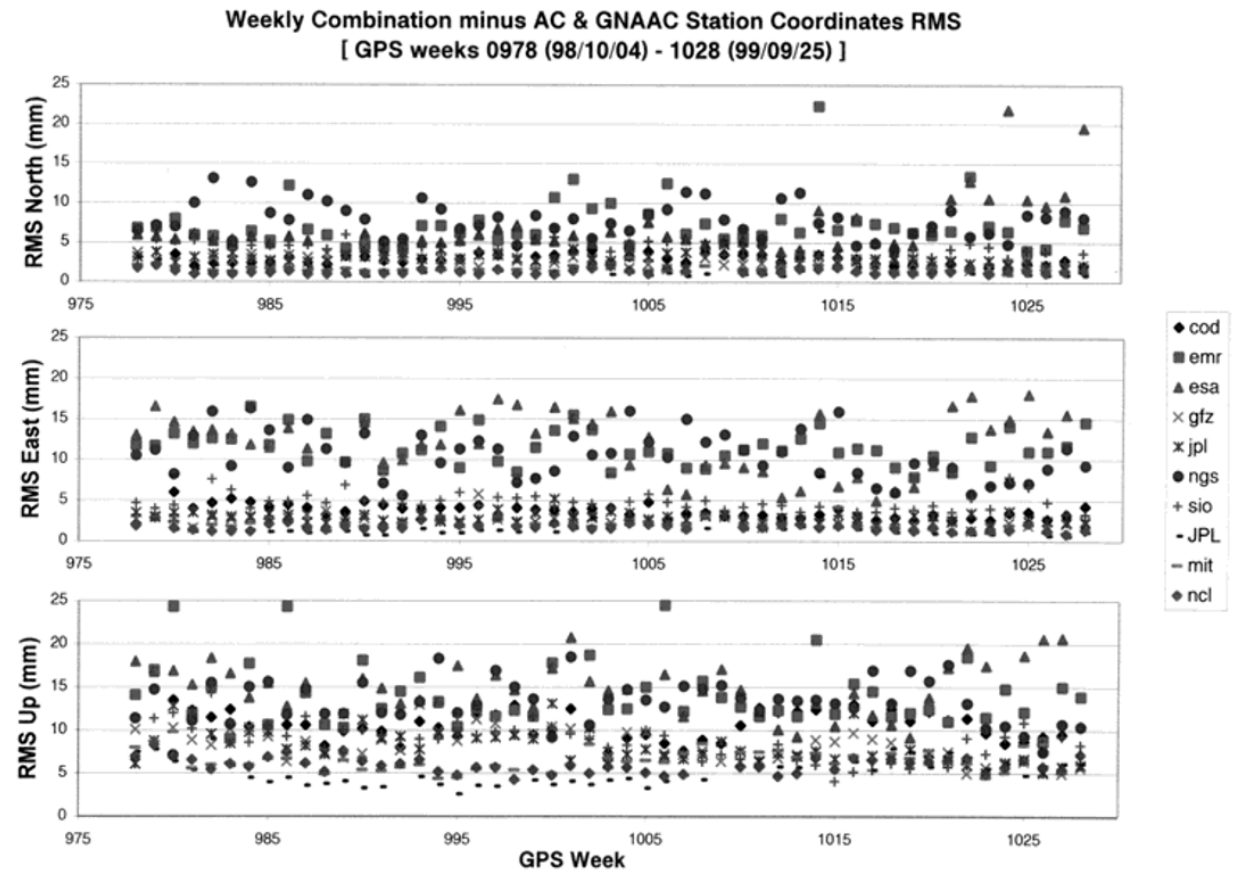

Fig. 3. RMS of North/East/Up Station Coordinate Differences between the AC and GNAAC and the "Weekly" Combination Solutions.

Cumulative Combination minus AC \& GNAAC Station Coordinates RMS [ GPS weeks $0978(98 / 10 / 04)$ - $1028(99 / 09 / 25)$ ]
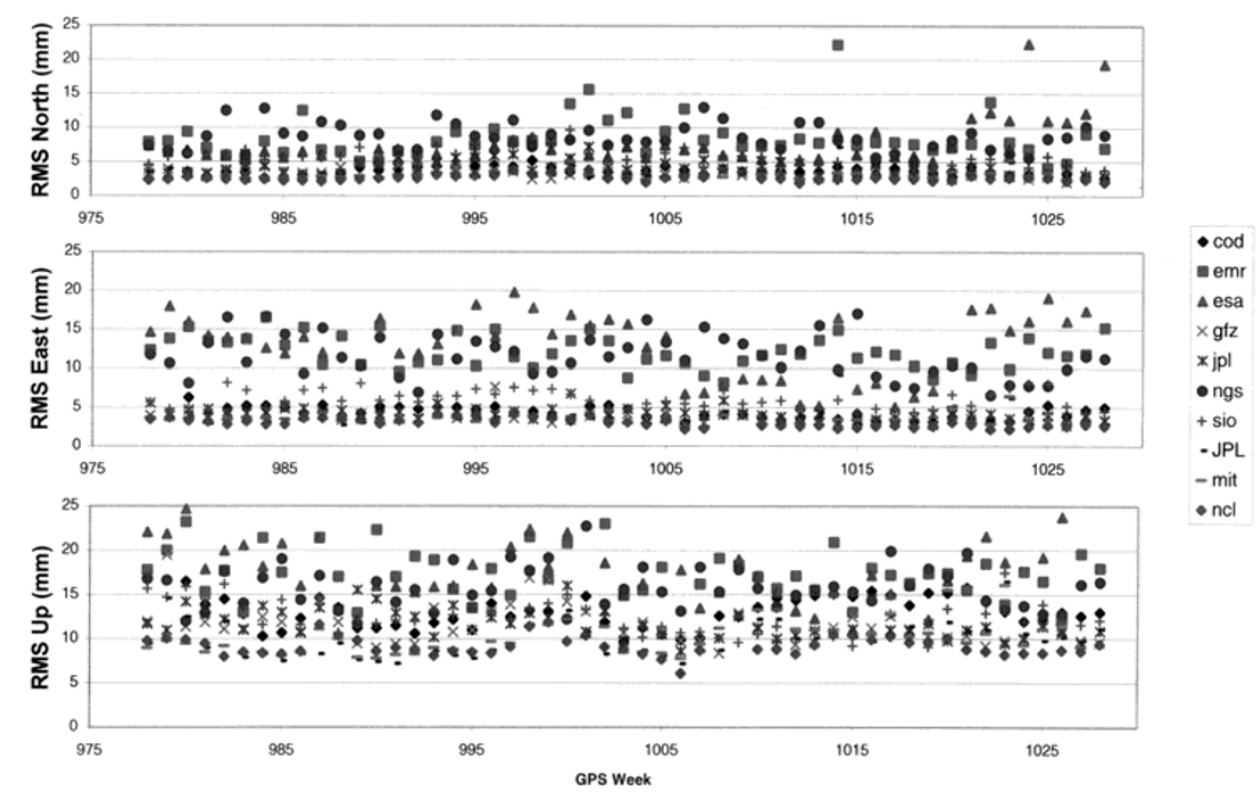

Fig. 4. RMS of North/East/Up Station Coordinate Differences between the AC and GNAAC and the Cumulative Combination Solutions.

and 7) the conflicts/inconsistencies.

\section{Results}

The results presented in this section are extracted from the weekly summary files between GPS weeks 0978 (October 4, 1998) to 1028 (September 25, 1999). Even though the solutions are publicly available only since GPS week 0999 (February 28, 1999), the procedure was tested with data dating back to GPS week 0978. Those test weeks are also included here.

Figure 1 shows the time series for the scale factors (square root of variance factors) that were applied to the $\mathrm{AC}$ and GNAAC weekly solutions. It is interesting to note the consistency of the scale factors. On average, the AC/GNAAC scale factors vary from week to week by about $15 \%$. In the best case (ncl), it is as low as $7 \%$. The first iteration of the combination takes advantage of this variance factor stability by using the previous week estimate. Under normal 
conditions, 2 to 3 iterations are sufficient for the scale factor estimation to converge. In most cases, the scale factor has random noise behavior. When all the AC started to use ITRF97 RF stations on GPS week 1021, steps were observed on some time series.

The weekly average residuals between the AC/GNAAC and ITRF for the RF stations is generally within 2-3 mm in all 3 directions (North, East, Up). The overall average is $(-0.5 \mathrm{~mm},-0.1 \mathrm{~mm}, 0.4 \mathrm{~mm})$. Weighted average time series are correlated and they show small systematic biases. The RMS of the residuals (Fig. 2) is about $(4.8 \mathrm{~mm}, 6.4 \mathrm{~mm}$, $12.4 \mathrm{~mm}$ ) for the RF stations. This shows that the alignment with ITRF is well within the computed RMS. The RMS of the residuals in the horizontal are stable, there seems to be a marginal improvement in the vertical axis.

Figure 3 shows the RMS for the AC/GNAAC weekly solutions with respect to the "weekly" combination solutions. It includes all the combined stations. The residuals with respect to the weekly combined solution have an overall weighted average of $(-0.4 \mathrm{~mm}, 0.1 \mathrm{~mm},-0.4 \mathrm{~mm})$. The weighted average is marginally better than when comparing the RF stations to ITRF. The RMS for each direction is (3.9 $\mathrm{mm}, 5.4 \mathrm{~mm}, 10.4 \mathrm{~mm})$. It is an indication of the internal consistency of the weekly AC/GNAAC station coordinates solutions. This also indicates that the RF stations coordinates and velocity estimates are consistent. Furthermore this also confirms the consistent quality of the horizontal station coordinates solutions and the marginal improvements in the vertical estimates. The best agreements are with the GNAAC solutions. They have the horizontal RMS at $1-2 \mathrm{~mm}$ and the vertical RMS at 4-6 $\mathrm{mm}$. This is an indication of the upper bound for the processing noise.

Figure 4 show the RMS between the AC/GNAAC weekly solutions and the cumulative combined solution at the weekly solution epoch. The comparison includes all the combined stations. The overall weighted average of the residuals is $(-1.2 \mathrm{~mm}, 0.0 \mathrm{~mm}, 1.3 \mathrm{~mm})$. It is larger than those estimated for the ITRF and the "weekly" combination comparisons. Some systematic effects present in the weekly and/or cumulative solution likely cause this. A close look at the residuals time series has confirmed the presence of annual and semi-annual periods at a number of stations, predominantly in the vertical component. Some stations also show more erratic behavior, which can reach several $\mathrm{mm}$. The RMS are, on the average, about $5.0 \mathrm{~mm}, 6.5 \mathrm{~mm}$ and 13.7 $\mathrm{mm}$. It is almost identical to the results obtained during the comparisons with the RF stations. It is an indirect indication of good internal consistency between the ITRF and the cumulative solutions. The ratio of the "weekly" and the above RMS is about 0.8 for both the horizontal and vertical components. Using the law of accidental error propagation one can estimate that about $60 \%$ of the noise is probably caused by the short term effects (about one week) and about $40 \%$ has a longer-term signature.

\section{References}

Boucher, C., Z. Altamimi, and P. Sillard (eds.), The 1997 International Terrestrial Reference Frame (ITRF97), IERS Technical Note 27, Observatoire de Paris, Paris, 1999.

DeMets, C., R. G. Gordon, D. F. Argus, and S. Stein, Effects of recent revisions to the geomagnetic reversal time scale on estimates of current plate motions, Geophys. Res. Lett., 21, 2191-2194, 1994.

Kouba, J., J. Ray, and M. M. Watkins, IGS reference frame realization, 1998 IGS Analysis Center Workshop Proceedings, edited by J. M. Dow et al., pp. 139-172, European Space Operations Center, Darmstadt, Germany, 1998

Vanicek, P. and E. Krakiwsky, Geodesy: The Concepts, 697 pp., NorthHolland Publishing Company, 1986.

R. Ferland (e-mail: ferland@geod.nrcan.gc.ca), J. Kouba (e-mail: kouba@geod.nrcan.gc.ca), and D. Hutchison 